http://jmscr.igmpublication.org/home/ ISSN (e)-2347-176x ISSN (p) 2455-0450 crossref DOI: https://dx.doi.org/10.18535/jmscr/v9i2.24

\title{
Metaplastic Breast Carcinoma: What First? Surgery or Adjuvant Therapy? Case Report and Literature Review
}

\author{
Authors \\ Aishwarya Mohan*, G.D. Bakhshi, Kanishk Patil, Md Furqan, \\ Urvashi Jain, Kushal Heroor
}

Department of General Surgery, Grant Govt. Medical College and Sir J.J. Group of Hospital, Mumbai, India *Corresponding Author

Aishwarya Mohan

Department of General Surgery , Grant Govt. Medical College and Sir J.J. Group of Hospital, Mumbai, India- 400008

Abstract
Breast cancer is the second most leading cause of female mortality. Early identification and classification is
crucial in deciding the plan of management. Metaplastic breast carcinoma (MBC) is a rare entity with no
standard treatment protocol yet established. We present a case of locally advanced metaplastic breast
carcinoma treated primarily with modified radical mastectomy (MRM). A brief case report and review of
literature is presented.
Keywords: Metaplastic breast carcinoma, ulcero-proliferative breast cancer, chemorefractory.

\section{Introduction}

Metaplastic breast carcinoma (MBC) is exceedingly rare with incidence of $0.2-5 \%{ }^{(1)}$ of all breast cancers. ${ }^{(2)}$ It is characterised by presence of two cellular types- epithelial and mesenchymal. World Health Organisation (WHO) classified it as a separate histological type in 2012. ${ }^{(3)}$ These cancers tend to be of triple negative phenotype (estrogen receptor, progesterone receptor and herceptin receptor negative), aggressive in nature, presenting with larger primary tumor size, less nodal involvement, chemoresistant and with decreased survival $^{(1)}$. They have a higher histological grade, P53 and Ki67 over expression, hence more chemorefractory. Patients are usually above 50 years, with 5 year disease survival rate according to Surveillance, Epidemiology and End Results Program (SEER) database to be $77 \%$ compared to $85 \%$ of a triple negative infiltrating duct carcinoma (IDC). ${ }^{(4)}$

\section{Case Report}

A 76 years old female presented with lump in her left breast since 1 year. She had 4 children who were adequately breastfed with first pregnancy at 20 years of age. She developed pain and redness over the lump 4 months back. She was treated by incision and drainage in a local hospital for the same following which she developed an ulcer which rapidly increased in size. She had no comorbidities and no family history of breast cancer. On examination she was tachycardic with 
left breast showing ulcero-proliferative mass measuring $10 \times 7 \mathrm{~cm}$ with destruction of the nipple areola complex (NAC) extending into the upper quadrant with foul smelling discharge (figure 1). Anterior group of axillary lymph node (LN) was palpable and there was no fixity to pectoralis major muscle. Her complete blood count showed leucocytosis of 40,000/ cmm. She was started with Intravenous antibiotics in view of infection.

Edge biopsy revealed infiltrating duct carcinoma (IDC) with ulceration of epidermis with dense inflammation. Computed Tomography (CT) scan of chest abdomen and pelvis showed ulceroproliferative mass over her left breast with single axillary node metastasis. (figure 2) There were no lung or liver metastasis. There was associated hiatus hernia leading to poor pulmonary reserve of the patient.( Positron Emission Tomography) PET CT showed 10.9 × 8.7 x $9.4 \mathrm{~cm}$ fluorodeoxy glucose (FDG) enhancing lesion involving whole left breast and NAC. Fat planes with pectoralis muscle appeared indistinct at few places $.3 .5 \times 3$ cm FDG enhancing left axillary Lymph nodes were seen (figure 3). There was no evidence of distant metastasis. In view of breast being focus of sepsis, she was worked up for surgery. Patient underwent modified radical mastectomy (Figure 4,5). She had uneventful post operative recovery. Her leucocyte count became normal and clinical condition improved. Final histopathology report of specimen showed metaplastic carcinoma with squamous differentiation. It had high nuclear grade, triple negative, with presence of lymphovascular emboli with one out of $11 \mathrm{LN}$ showed metastasis. Resection margins were negative. Patient was advised chemoradiation, however, patient declined to take any adjuvant therapy. Follow up of 2 months has shown her to be disease and symptom free.

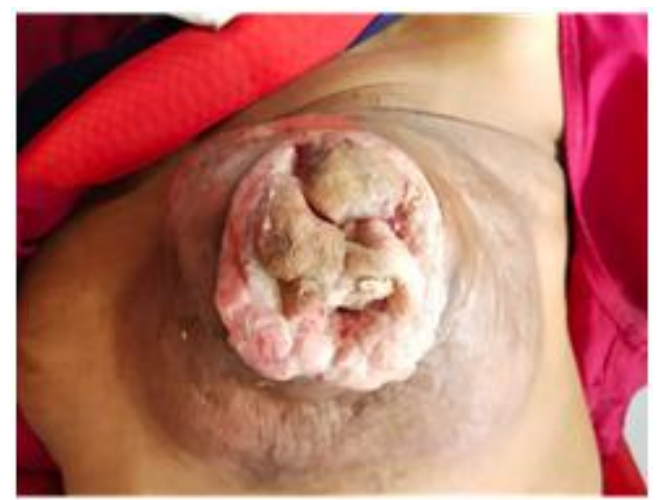

Figure 1: Left breast showing breast showing ulcero-proliferative mass measuring $10 \times 7 \mathrm{~cm}$ with destruction of the nipple areola complex (NAC)

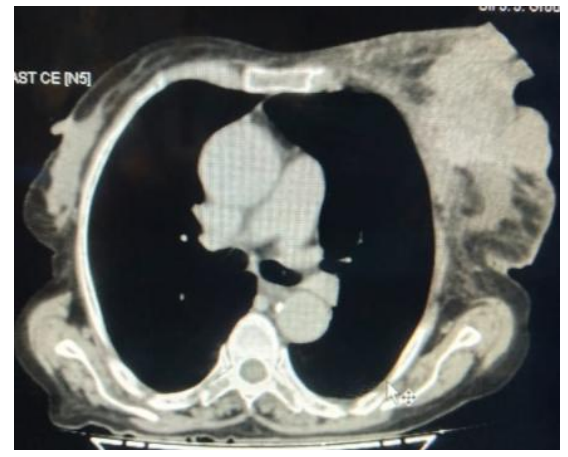

Figure 2 CT scan showing ulcero-proliferative mass over her left breast with single axillary node metastasis and loss of fat planes above muscle.

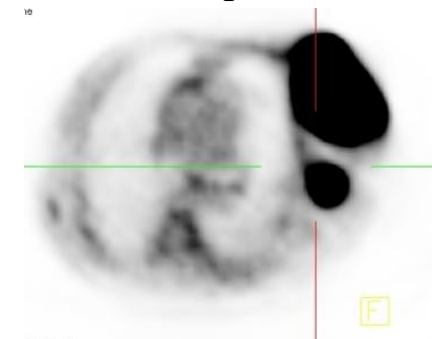

Figure 3: Pet CT showing left breast mass fat planes with pectoralis muscle indistinct at few places and $3.5 \times 3 \mathrm{~cm}$ FDG enhancing left axillary lymph nodes.

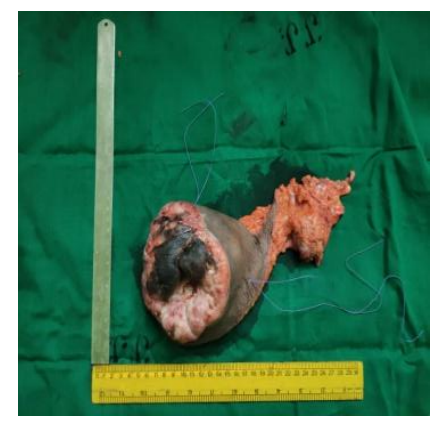

Figure 4: Specimen of Mastectomy 


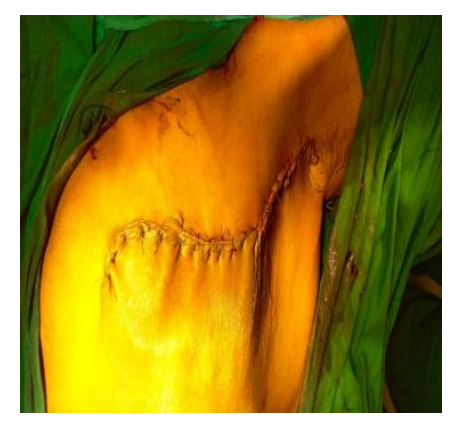

Figure 5. Post operative suture line.

\section{Discussion}

In a study involving Indian tertiary care centre data incidence of metaplastic breast cancer was $0.5 \%$ (31/6180). ${ }^{(5)} \mathrm{MBC}$ usually presents as lump accompanied by inflammatory signs like ulceration, redness and discharge ${ }^{(6)}$. Present case presented with inflammed, infected and ulcerative mass. According to one study ${ }^{(5)}$, the most common histopathological differentiation was squamous (45.16\%), followed by sarcomatoid histology $(32.25 \%)$ and last two being chondroid (9.68\%) and mixed histology (12.9\%). In present case patient had squamous differentiation. Factors in favour of metaplastic breast cancer are older age of presentation, rapid growth and larger primary tumor size and less distant metastasis. Present case satisfied all except the node positivity status. High clinical acumen is thus needed to decide mastectomy vs neoadjuvant therapy in such cases. Management is also affected by factors like disseminated cancer, physical deformity, foul odour, bleeding, infection and psychosocial factors in cases of locally advanced breast cancer (LABC). Infection and physical condition of the patient was a contributing factor for decision of modified radical mastectomy in present case.

Chances of metaplastic breast cancer diagnosed preoperatively is dismal with diagnostic rate of MBC with core needle biopsy as low as $40 \%$ with $60 \%$ being diagnosed as ordinary infiltrating duct carcinoma $^{(7)}$. Consistent with this was our finding in which the initial core biopsy showed IDC which was postoperatively found to be metaplastic breast cancer. Accurate preoperative diagnosis is important as $\mathrm{MCB}$ is unlikely to respond to chemotherapy and given its rapid growth could become inoperable in duration of few months. In present case MBC was not the driving factor but patients sepsis governed the decision of surgery.

Due to rarity of the disease and lack of standard treatment protocols options available are:

1)- Neoadjuvant Chemotherapy - this has shown poor response and high failure rate in triple negative $\mathrm{MBC}^{(8)}$

2)- Mastectomy- this is the most commonly used treatment based on retrospective studies and the one used in this case scenario.

3)- Postoperative Radiotherapy: This has shown survival benefit but enough prospective studies are yet to be carried out for its standardisation. Result of tseng et al. analysis utilysing the SEER database suggested that adjuvant radiation improved both overall and disease specific survival for all patients undergoing treatment for $\mathrm{MBC}^{(9)}$. Patients receiving radiotherapy (RT) demonstrated $36 \%$ and $26 \%$ decreases in death from any cause and breast related mortality, respectively. Postmastectomy RT has a more limited role. In this setting, RT is recommended to patients with 4 or more metastatic axillary nodes, large $>/=5 \mathrm{~cm}$ primary tumors and chest wall invasion. Tseng et al described mastectomy patients who received RT showing 33\% decreased risk of death from any cause, pts with $>/=5 \mathrm{~cm}$, or 4 or more nodes derived $47 \%$ and $42 \%$ decreased risk of death from all cause and breast related mortality. Hence RT can be considered for patients with advanced features after mastectomy. ${ }^{(9)}$, In present case though patient was advised adjuvant chemoradiation, however, she refused the same inspite of counselling.

High volume centres can promote patients with advanced disease to enroll in clinical trials, to help research in chemotherapy and immunotherapy.

\section{Conclusion}

Metaplastic breast cancer is rare, heterogeneous, aggressive, most commonly triple negative cancer. It is usually chemorefractory, and infected cases 


\section{JMSCR Vol||09||Issue||02||Page 155-158||February}

need early mastectomy. Use of radiation as adjuvant therapy for advanced cases may improve overall survival. Research is needed in field of chemotherapy and immunotherapy directed against heterogeneous genetic profile of the disease.

\section{References}

1. Reddy TP, Rosato RR et al. A comprehensive overview of metaplastic breast cancer: clinical features and molecular aberrations. Breast Cancer Research,2020; 22,121

2. Lakhani SR, Ellis IO et al. (2012) WHO classification of tumors of the breast. World Health Organization classification of tumours. (4th edn). Lyon: IARC Press, USA.

3. Ghosh M, Muneer A et al. Metaplastic carcinoma breast: A clinical analysis of nine cases. J Clin Diagnostic Res. 2017; 11:XR01-XR03

4. Nelson RA, Guye ML, Luu T, Lai LL. Survival Outcomes of Metaplastic Breast Cancer Patients: Results from a US Population-based Analysis. Ann Surg Oncol. 2015; 22:24-31

5. Lalnun puii et al. Survival outcomes of metaplastic breast carcinoma: An Indian tertiary care centre data. J Clin Oncol. 2020; 38.15_suppl.e12579

6. Kuo, S. H., Chen, C. L. et al. Metaplastic carcinoma of the breast-analysis of eight Asian patients with special emphasis on two unusual cases presenting with inflammatory-type breast cancer. Anticancer Research, 2000;20: 2219-22.

7. Takahashi K. A rare case of metaplastic carcinoma of the breast that could be diagnosed with preoperative core needle biopsy and the improbability of skin invasion. Int J Surg Open. 2019; 16:29-33
8. Chen IC, Lin $\mathrm{CH}$, Huang $\mathrm{CS}$, Lien $\mathrm{HC}$, Hsu C, Kuo WH, et al. Lack of efficacy to systemic chemotherapy for treatment of metaplastic carcinoma of the breast in the modern era. Breast Cancer Res Treat. 2011; 130:345

9. Tseng WH, Martinez SR Metaplastic breast cancer: to radiate or not to radiate? Ann Surg Oncol 2011;18: 94-103. 